\title{
A PROGRAM FOR CALCULATING AND PLOTTING SOFT X-RAY OPTICAL INTERACTION COEFFICIENTS FOR MOLECULES
}

M. M. Thomas, J. C. Davis, C. J. Jacobsen, and R.C.C. Perera Center for $\mathrm{X}$-ray Optics, Acceleration and Fusion Research Division, Lawrence Berkeley Laboratory, Berkeley, CA

Comprehensive tables for atomic scattering factor components, $f 1$ and $f 2$, were compiled by Henke et al. for the extended photon region of $50-10000 \mathrm{eV}$. Accurate calculations of optical interaction coefficients for absorption, reflection and scattering by material systems (e.g. filters, multi-layers, etc...), which have widespread application, can be based simply upon the atomic scattering factors for the elements comprising the material, except near the absorption threshold energies. These calculations based upon the weighted sum of $f 1$ and $f 2$ for each atomic species present can be very tedious if done by hand. This led us to develop a user friendly program to perform these calculations on an IBM PC or compatible computer. By entering the chemical formula, density and thickness of up to six molecules, values of the f1, f2, mass absorption, transmission efficiencies, attenuation lengths, mirror reflectivities and complex indices of refraction can be calculated and plotted as a function of energy or wavelength. This program will be available for distribution.

\section{DISCLAIMER}

This report was prepared as an account of work sponsored by an agency of the United Statcs Government. Neither the United States Govetnment nor any agency thereof, nor any of their employees, makes any warranty, express or implied, o: assumes any legal liability or responsibility for the accuracy, completencss, or usefulness of any information, apparatus, product, or process disclosed, or represents that its use would not infringe privately owned rights. Reference herein to any specific commercial prodisct. process, or service by trade name, trademark. manufacturer, or otherwise does not necessarily constitute or imply its endorsement, recommendation, or favoring by the United States Government or any agency thereof. The views and opinions of authors expressed herein do not nocessarily state or reflect those of the United States Government or any agency thereor. 


\section{Introduction}

With the tabulation of the elemental scattering factors $\left(f_{1}\right.$ and $\left.f_{2}\right)$ by Henke et al. 1,2 their use in designing optical $x$-ray elements has become more routine. Transmission and absorption of filters and reflectivity from $x$-ray mirrors in the 10 $\mathrm{eV}$ to $10 \mathrm{keV}$ energy range are calculated with these tabulated values. When, however, analyzing systems comprised of molecules or mixtures, or if a large number of values are desired over a range of energies, the calculations can become tedious. This program, Optical Constants Grapher (OCG), was written to address these problems by doing calculations from the tabulated $f_{1}, f_{2}$ values and quickly displaying thers graphically or in a table for the user. OCG has the following primary features:

1) Calculates the following "constants" in the $10 \mathrm{eV}-10$ keV range: Absorption Lergth, Transmission Efficiency, Mass Absorption Coefficient, Reflectivity at fixed angle, Reflectivity at fixed energy.

2) Graphically displays above constants (and f1, f2) over any specified energy range, linearly or in log-log format.

3) Displays above constants plus delta and beta (defined in the theory section) for a specific energy in a tabular format.

4) Facilitates comparisons of different materials by keeping a running list of up to six molecules - the constants $\mathrm{of}_{\mathrm{f}}$ which are graphed simultaneously.

5) Can make a multi-column file containing a desired constant for all the molecules in the list over the complete energy range.

6) Alls.rs user to work in units of energy (eV) or of wavelength (Angstroms).

\section{A. System Requirements}

OCG runs on IBM PC computers and compatibles with at least 640k RAM. An EGA card set for color output and a color monitor are also required for graphical output. A hard disk drive is highly desirable but not required. Also note that the print screen function will not operate under DOS 3.30 due to bugs in the system software. It is suggested that if printed output from OCG is required, install the program on a machine operating under DOS 3.20 .

\section{Theory}

The photo-absorption cross-sections ( $\mu$ - from which $f_{1}$ and $f_{2}$ were derived) are based upon the best currently available experimental data as well as the theoretical values calculated by G. Doolen and D. Liberman ${ }^{3}$. 
No attempt was made to determine near edge structure since near edge data is representative of a given chemical environment, and currently available theory is not capable of modelling such collective effects. The current fits are extrapolated linearly in $\log \mu, \log E$ space up to the edge as given in Bearden and Burr ${ }^{4}$. Detailed discription of deriving $f_{1}$ and $f_{2}$ values is presented elsewhere ${ }^{1,2}$.

The actual range of the data in the elemental scattering factor files included with OCG is as follows: $f_{1}$ extends from $50 \mathrm{eV}$ to $10 \mathrm{keV}$ while $f_{2}$ extends from $10 \mathrm{eV}$ to $10 \mathrm{keV}$. The $\mathrm{f}_{1}$ is derived from a Kramer's-Kronig integral:

$$
f_{1}(E)=Z+\frac{1}{\pi r_{0} h c} \int_{0}^{\infty} \frac{\varepsilon^{2} \mu_{a}(\varepsilon) d \varepsilon}{E^{2}-\varepsilon^{2}}-\Delta f
$$

where $\Delta f_{r}$ is the relativistic correction as discussed in Cromer and Liberman ${ }^{5}$, and in Jensen ${ }^{6}$. As for $f_{2}$, the optical theorum gives:

$$
f_{2}(E)=\frac{1}{2} \frac{1}{r_{0} h c} E \mu_{a}(E)
$$

Fig. 1 illustrates both the $f_{1}, f_{2}$ derived from experimental $\mu$-values of carbon ${ }^{7}$ and the corresponding fitted scattering factors ${ }^{1,2}$. Note that only the overall features of the edge structure are maintained in the fit while all near edge effects are neglected. Hence, constants plotted in OCG near edges should be treated skeptically.

\section{A. Calculating Molecular $\mathbf{f}_{\mathbf{1}}$ and $\mathbf{f}_{\mathbf{2}}$}

Molecules and mixtures are treated as mixed gases of their elemental components. That is to say, molecular geometry and molecular orbitals are not considered. In calculating the average scattering factor, each element contributes according to its numerical proportion.

Calculating the average scattering factors for molecules and mixtures is accomplished as follows:

$$
\overline{n f_{1}}=\sum_{q} n_{q} f_{1 q} \quad \overline{n f_{2}}=\sum_{q} n_{q} f_{2 q}
$$

where $n_{\mathrm{q}}$ is the number of atoms per $\mathrm{cm}^{3}$ of type-q.

Note that the $f_{1}$ and $f_{2}$ values plotted and tabulated with OCG are the above $\bar{n}_{x}^{-}$divided by the number of molecules $/ \mathrm{cm}^{3}$. They are the average scattering factor per chemical unit (molecule, unit of mixture, etc...), not the average scattering factor per unit volume as calculated above. 
Figure 1
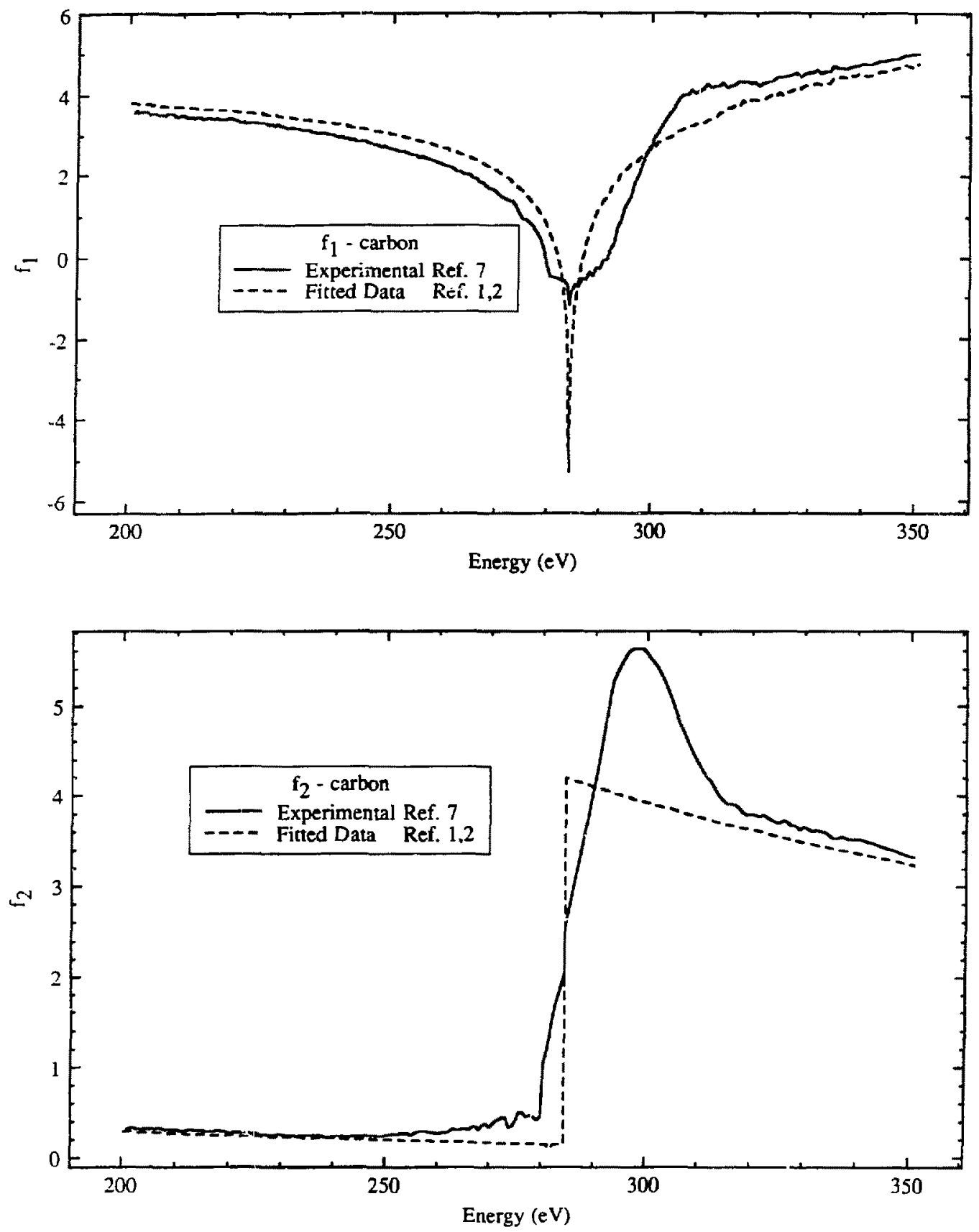


\section{B. Optical Constants used in OCG}

Listed below are the formulae of the optical constants used in OCG.

Definitions of symbols are as follows: $t$ is the thickness of sample; $r_{0}$ is the classical electron radius $\left(e^{2 / m c^{2}}\right) ; \lambda$ is the wavelength; $\overline{n f_{x}}$ is the average $f_{x}$ value for one $\mathrm{cm}^{3}$ of sample; $\xi$ is the density $\left(\mathrm{gm} / \mathrm{cm}^{3}\right) ; \theta$ is the grazing angle.

For $x$-rays, the complex index of refraction is written $\hat{n}=1-\delta$-i $\beta$ where $\delta=\frac{r_{0} \lambda^{2}}{2 \pi} \overline{n f_{1}}$ and $\beta=\frac{r_{0} \lambda^{2}}{2 \pi} \overline{n f_{2}}$, when $\delta$ and $\beta$ are small positive quantities.

$$
\begin{gathered}
\text { Transmission Efficiency }=t^{-2 / r_{0} \lambda \overline{n f_{2}}} \\
\text { Absorption Length }=\left[2 r_{0} \lambda \overline{n f_{2}}\right]^{-1} \text { results in microns } \\
\text { Mass Absorption Coefficient }=\frac{2 r_{0} \lambda \overline{n f_{2}}}{\xi} \\
\text { Reflectivity }=\frac{1}{2}\left[\frac{4 \rho^{2}(\sin \theta-\rho)^{2}+4 \beta^{2}}{4 \rho^{2}(\sin \theta+\rho)^{2}+4 \beta^{2}}\right]\left[1+\frac{4 \rho^{2}(\rho-\cos \theta \cot \theta)^{2}+2 \beta}{4 \rho^{2}(\rho+\cos \theta \cot \theta)^{2}+2 \beta}\right] \\
\text { where, } \rho^{2}=\frac{1}{2}\left\{\sin ^{2} \theta-2 \delta+\left[\left(\sin ^{2} \theta-2 \delta\right)^{2}+4 \beta^{2}\right]\right\}
\end{gathered}
$$

Instructions for using OCG are found in Appendices A and B below. Appendix $A$ contains the procedure for installation as well as a tutorial to get the user started. Appendix B contains a detailed description of all the menu items and commands available in OCG. 


\section{Appendix A: Instructions}

\section{Conventions used in Manual}

For clarity the following conventions will be followed in the manual. 1) As all features of the program allow the user to input responses in terms of both energy and wavelength, instructions in this write-up will only refer to energy inputs. It is understood that the user can run the program in either energy or wavelength mode as described in Appendix B. 2) The values f1, $\mathrm{f} 2$ and the calculations based on them are referred to as "constants" to generalize the instructions.

\section{Installation}

The program and data are contained on two $360 \mathrm{k}$ floppy diskettes. Initially make copies of these diskettes and write-protect the masters if they are not already. Note: the program cannot run successfully if the disks you are using are writeprotected.

\section{Hard Disk Drive Installation:}

Copy all of the files on the program disk to some new subdirectory on your hard disk. As an example I'll use "SF". Now that all the files have been copied into "C: $\backslash S F \backslash$ ", create a subdirectory within the program directory to hold the data files; I'1] use "SFDATA". Copy all the data files into that subdirectory - e.g. "C:\SF\SFDATA\".

Now, run the program by typing "SF[return]". If all is well, you should see something to the effect:

$$
\begin{aligned}
& \text { The parameter file was not found (etc...) } \\
& \text { Are you using a floppy or a hard disk drive ( } F \text { or } H) \text { ? }
\end{aligned}
$$

Type " $\mathrm{H}$ " and then when prompted, enter the full path to the data. In our example, you would use "C: \SF\SFDATA $\backslash$ ". REMEMBER THE LAST BACKSLASH. DOS is not very forgiving of mistakes in path structure and the program could end up thinking the data is not where you've put it.

The program has now created a small file in the program directory "SFPARM.DAT" which contains the path name to the data. If you ever make copies of this program to be placed on another machine, make sure this file is NOT included in the directory as the new machine will attempt to read the data from the pathname stored in this file which may not correspond to the new setup.

That's it. If run from the program directory, the program should from now on bypass the installation procedure and move right on to the rest of the program.

Floppy Disk Drive Installation: 
Using the non-write-protected copies you've made, insert the program disk into one of your floppy drives and type "SF". If all is well, you should see something to the effect:

\section{The parameter file was not found (etc...) \\ Are you using a floppy or a hard disk drive ( $\mathrm{F}$ or $\mathrm{H}$ ) ?}

Type " $\mathrm{F}$ " and then at prompt, enter the drive that you intend to read data from - e.g. "B". The computer will now create a small file called "SFPARM.DAT" on the program disk which will store which drive you specified and the fact that you are using a floppy system. If you ever make copies of the program disk for distribution, remove the "SFPARM.DAT" file for the new user will probably have a different system configuration than yours.

The computer should now respond with something like:

\section{PLEASE INSERT THE DATA DISK INTO DRIVE B:}

Do so, and press enter. From now on when "SF" is run from the program disk, the above line will appear, bypassing the installation procedure. From now on the drive specified above will be your data drive. If the program is running, the data diskette must be in that drive.

Overview

Once OCG is successfully running, the screen will clear and and the primary menu will appear. To obtain help from the program, hit the F1 key. On the left side of the screen is the main menu consisting of six items that will be explained later. On the right side of the screen is the Molecule List. It displays all those elements, molecules and compounds that have been entered into OCG. When a constant is graphed, the graph of the constant for each molecule in the list will be displayed simultaneously. Thus the Molecule List can be thought of as the current list of molecules being compared.

Since OCG was designed for occasional quick reference, its user interface was made to be as intuitive as possible. To accomplish this, the user interface relies heavily on arrow keys and special "hot-keys". Menu items can be selected either by moving the cursor (the cursor highlights an item in magenta) to the desired item and typing return, or be hitting the designated "hot-key" for the item (the single highlighted character in the item name).

\section{Graphing Constants}

Selecting Molecule List from the main menu allows the user to enter molecules and compounds into the list. Note that a cursor appears in the top "slot" of the list. Other slots may be selected by using the up and down arrow keys. This way, up to six molecules may be placed in the list.

To enter a molecule into the list, simply type its chemical formula in standard format. (Remember that OCG is case sensititive in order to distinguish between all 
92 elements - so beginning a chemical formula with a lower case letter will not produce any results.) Nested parentheses and suffixed multipliers (both integer and

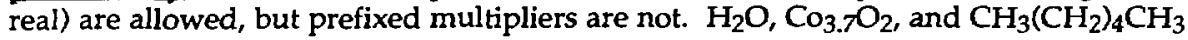
are examples of permitted chemical formulae, while $3 \mathrm{CO}_{2}$ is not because of the prefixed multiplier. Hit the return key and then enter the density $\left(\mathrm{gm} / \mathrm{cm}^{3}\right)$ and thickness of your sample in microns. Try entering a test molecule. Hitting return once more leaves the Molecule List.

Now, select the graph item from the main menu. A "pop-up" menu will appear with a list of the constants available to be graphed. For example, select the item F1 from the menu by typing ' 1 '. When the computer finishes its calculations, it will ask for the domain over which to graph the data. Type return to select the default values given in the brackets. Do the same for the range of the constant values. A graph will be created with a title (in this case "f1 vs. energy") and colored graph identifiers at the bottom containing the chemical formula of the compound and a number.

The color of the identifier corresponds, of course, to the color of the graph for that compound. The number below the chemical formula corresponds to different things depending on the constant being graphed. For $\mathrm{f} 1, \mathrm{f} 2$, transmission efficiency, absorption length, and mass absorption coefficient it is the thickness of the sample. For reflectivity at fixed angle, it is that angle, and for reflectivity at fixed energy it is that energy.

When you are finished looking at the graph, type return to continue. If you wish to see another view of the same data, type ' $y$ ' in response to the next question.

\section{Obtaining constants for a specific energy or wavelength}

With OCG you have the option of obtaining a table of constants for a specific molecule and energy. This is contained within the EXAMINE feature in the main menu. OCG must first, however, know which molecule in the list you wish to examine. Select MOLECULE LIST from the main menu. Place the cursor on top of the molecule you wish to examine and hit return. Typing return leaves the list, and also highlights the last molecule the cursor rested on. This highlighted molecule will be the one EXAMINE uses in its calculations.

Now select EXAMINE from the list. Enter an energy in $\mathrm{eV}$. The available constants will be displayed next to their labels. To view the constants for a different energy, merely enter that new energy. To leave EXAMINE, type return without entering an energy value.

If you wish to work in wavelength instead of energy while in EXAMINE and have not previously switched to wavelength, you may do so here. Simply type a CTRL-A without a return and the "energy" label should change to "wavelength" and the value next to the energy (if any) will be converted to Angstroms. Another Ctrl-A will do the reverse. Now just enter your responses in Angstroms - the other parts of the program will correspondingly request for angstroms instead of $\mathrm{eV}$ until you switch back to energy input.

\section{Saving Constants to a file}

For further calculations or plotting, it may be desirable to save a constant to a file. This is, of course, the function of the SAVE TO FILE menu item. It works 
exactly as does the GRAPH pop-up menu with the exception that it does not query for a domain or range, as the entire data set will be saved to disk.

The file created is a TEXT file using the floating point format of the C 'fprintf' function. The first column is the energy with the remaining columns

corresponding to each molecule in the list from top to bottom. 


\section{Appendix B: Reference}

This reference section contains summaries of each menu item in OCG. The procedure outlined above for graphing a constant should be enough to make the reader reasonably proficient using the program - this section is intended as a more complete reference. Note that for quick reference, the F1 key on the IBM will display a help sheet.

\section{Molecule List}

As demonstrated above, this command activates the molecule list to the right of the screen. Use the up and down arrow keys to move from one slot to the next. Chemical formulae may include parentheses and suffixed numerical multipliers (both integer and real) but not prefixed multipliers. Typing Ctrl-E while the cursor rests upon an existent molecule will allow the user to edit that molecules density and thickness data. Note that the data must be retyped - no short cuts exist for selecting existing data.

Typing delete or backspace while the cursor rests on a molecule will remove that molecule from the list. Typing return anywhere in the list without beginning a molecule first, exits the Molecule List. Exiting while the cursor resides on top of an existent molecule highlights that molecule, making it accessible via the EXAMINE function.

\section{Examine}

The EXAMINE feature tabulates the following constants: $f_{1}, f_{2}$, delta, beta, Transmission Efficiency, Attenuation Length, and Mass Absorption Coefficient. The formulae for these constants can be found in the Theory section above.

The currently highlighted molecule in the molecule list is selected as the molecule to be examined (see Molecule List section above). It's name appears at the top of the window. To see the constants at a particular energy (greater than $10 \mathrm{eV}$ and less than $10 \mathrm{k} \mathrm{eV}$ ) simply type that energy and hit return. The constants will be displayed next to their labels. OCG uses a simple linear interpoiation betweer the data points in its $f_{1}, f_{2}$, files. To see another set of constants, simply enter a different energy value.

Typing Ctrl-A toggles between energy and wavelength input. Typing Return or Escape before having begun typing an energy value exits the EXAMINE routine.

\section{Graph}

OCG graphs the following constants: $f_{1}, f_{2}$, Transmission Efficiency, Absorption Length, Mass Absorption Coefficient, Reflectivity - P(\%) - at fixed angle, and Reflectivity at fixed Energy. Each constant has an item in the graph submenu. The proper range of these constants is associated with the scattering factor that they depend upon. For example, constants that depend upon $f_{1}$ are only valid down to 50 $\mathrm{eV}$ whereas constants depending only on $\mathrm{f}_{2}$ are valid till $10 \mathrm{eV}$.

in the graph submenu there is also an item labeled 'Plot Parameters'. This selects whether the plot will be done with respect to energy or wavelength, and if it will be linearly or logarithmically scaled.

Changing the Plot Parameters 
Select Plot Parameters in the usual manner. At the first prompt, type 'A' for angstroms or ' $\mathrm{E}$ ' for $\mathrm{eV}$. At the second prompt, type ' $\mathrm{L}$ ' for linear or ' $\mathrm{G}$ ' for logarithmic. These selections will remain for all graphs until the user changes them again.

\section{$f_{1}, f_{2}$, Trans. Eff. , Abs. Length, Mass Abs. Coef.}

The graphs of these constants all work essentially the same way. The user selects the constant from the graph submenu, then enters the domain (in energy or wavelengths) and the range (of the constant being graphed). For linear graphs, domain and range are entered as MIN, MAX, TICKCOUNTS [return]. Tickcounts corresponds to the number of major divisions of the axis in question. For example, choosing an input of "0, 300, 3 [return]" would produce a graph that extended from 0 to 300 , with major iickmarks at 100 and 200 ( 3 major divisions) and minor tickmarks at 50, 150, and 250. 0 and 300 are not marked because they reside at the edge of the box which contains the graph. Note: the user may select a domain or range beyond the limits of the data set - say to improve the appearance of the graph. Also, note that initiaily the computer will give what it thinks are the best limits to contain all of the data. To choose these limits, simply type return instead of entering limits yourself.

For logarithmic graphs, there are a few differences. Note that the TICKCOUNTS are not requested from the user - the computer supplies this. Also, the exact limits requested will not be those used for the graph. The computer will pick limits just outside of those given so that tickcounts will reside on powers of 2 , 5 , and 10.

As mentioned above, the value listed below the chemical formulae in the graph produced is the thickness of the sample.

Finally, the computer $u$ ill remember the limits you give it for a particular type of graph. Say you are using linear scaling and working in $\mathrm{eV}$; for any future linear/eV graph the limits you seiected will become the default limits.

Reflectivity at Fixed Angle

This constant is listed as "P(\%) Fixed mRad" in the graph submenu. Upon selection it asks for the constant grazing angle to be used, with a default of 10 milliradians. DO NOT enter 0 here. An angle for each molecule in the list must be entered. Subsequently it asks for the domain and range of the graph in the usual manner.

The values listed below the chemical formulae in the graph refer to the fixed angles used.

\section{Reflectivity at Fixed Energy}

This constant is listed as "P(\%) Fixed Energy". As usual, although energy is listed in the title, this function may be performed in wavelengths as well. The computer first queries for the constant energy. As the Reflectivity uses $f 1$ and the $f 1$ files do not extend below $50 \mathrm{eV}$ a response greater than $50 \mathrm{eV}$ (or less than 248 Angstroms) is required. Again, an energy for eacn molecule must be entered.

The next query asks for the limits in miliiradiaiss of the data to be calculated. This query is unusual in that it will specify rot the domain of the graph, but the domain of the data calculated. Again, the lower limit must not be less than or equal to zero. After this, the usual domain and range prompts for the graph itself appear. 
Here, the values below the chernical formulae in the graph refer to the fixed energies used.

\section{Save to File}

Saving a constant to a file is performed the same way as graphing a file. The only difference in the queries are that no domain and range information for the graph itself is requested, as all of the available data will be saved to the disk. As for the graph, where the constant is graphed for each molecule in the list, the file created will have a column for each molecule in the list - ordered from to bottom. The first column lists the energies, wavelengths or angles that serve as the domain of the data. The remaining columns correspond to the molecules in the list.

\section{Extra Hot-Keys}

There are a few extra hot-keys that don't correspond directly to menu-items available to the user. Most of these have been mentioned previously.

Pressing F1 at any point in the main menu or in the Molecule List will call up the help sheet. It is intended only as a short summary of the features of OCG.

Pressing Ctrl-A while in the main rnenu or while in the Examine feature toggles between energy and wavelength input and display.

Pressing Ctrl-E while in the Molecule List and while the cursor resides on an existent molecule, edits the density and thickness information for that molecule.

Pressing Escape in most parts of the program will return control to the main menu. 


\section{Acknowledgements}

This work was supported by the Director, Office of Energy Research, Office of Basic Energy Sciences, Materials Sciences Division, of the U.S. Department of Energy under Contract No. DE-AC03-76SF0C098 and by the Air Force Office of Scientific Research, of the U.S. Department of Defense under Contract No. F49620-87-K-0001. 


\footnotetext{
1 B.L. Henke, P. Lee, T.J. Tanaka, R.L. Shimabukuro, and B.K. Fujikawa, Atomic Data and Nuclear Data Tables 27, 1 (1982)

2 B.L. Henke, J.C. Davis, E.M. Gullikson, and R.C.C Perera, Lawrence Berkeley Laboratory, Berkeiey, CA. LBL-26259 UC-411 (1988)

3 G. Doolen and D. Liberman, Physica Scripta 36, 77 (1987)

4 J.A. Bearden and A.F. Burr, Rev. Mod Phys. 39, 125 (1967)

5 D. T. Cromer and D. Liberman, J. Chem. Phys. B. 53, 189i (1970)

6 M.S. Jensen, Phys. Lett. A 74, 41 (1979)

7 N.K. Del Grande, K.G. Tirsell, M.G. Schneider, R.F. Garrett, E.M. Kneedler, and S.T. Manson, J. de Phys. Colloque C9, Supp. 12, Tome 48, (1987)
} 